\title{
The Short-Run and Long-Run Effects of Behavioral Interventions: Experimental Evidence from Energy Conservation
}

\author{
Hunt Allcott and Todd Rogers*
}

January 20, 2014

\begin{abstract}
We document three remarkable features of the Opower program, in which social comparisonbased home energy reports are repeatedly mailed to more than six million households nationwide. First, initial reports cause high-frequency "action and backsliding," but these cycles attenuate over time. Second, if reports are discontinued after two years, effects are relatively persistent, decaying at 10-20 percent per year. Third, consumers are slow to habituate: they continue to respond to repeated treatment even after two years. We show that the previous conservative assumptions about post-intervention persistence had dramatically understated cost effectiveness and illustrate how empirical estimates can optimize program design.
\end{abstract}

JEL Codes: D03, D12, L94, Q41

We thank Ken Agnew, David Cesarini, Gary Charness, Paul Cheshire, Lucas Davis, Stefano DellaVigna, Xavier Gabaix, Francesca Gino, Uri Gneezy, Michael Greenstone, Judd Kessler, David Laibson, Katy Milkman, Sendhil Mullainathan, Karen Palmer, Charlie Sprenger, staff at the utilities we study, and a number of seminar participants for feedback and helpful conversations. Thanks also to Tyler Curtis, Lisa Danz, Rachel Gold, Arkadi Gerney, Marc Laitin, Laura Lewellyn, Elena Washington, and many others at Opower for sharing data and insight with us. We are grateful to the Sloan Foundation for financial support of our research on the economics of energy efficiency. Stata code for replicating the analysis is available from Hunt Allcott's website.

Opower provided the data analyzed in this paper to the authors under a nondisclosure agreement. The authors and Opower structured the agreement in a way that maintains the authors' independence. In particular, the agreement stipulates that Opower has the right to review the publication prior to public release solely for factual accuracy.

\footnotetext{
*Allcott: NYU Department of Economics. hunt.allcott@nyu.edu. Rogers: Harvard Kennedy School.
} todd_rogers@hks.harvard.edu. 
Appendix: For Online Publication

The Short-Run and Long-Run Effects of Behavioral Interventions: Experimental Evidence from Energy Conservation

Hunt Allcott and Todd Rogers 


\section{Appendix I: Surveys of Self-Reported Actions}

During the past three years, Opower has surveyed about six thousand people in treatment and control groups in six sites nationwide, including 800 people in Site 2. These are telephone surveys, and completion rates are typically between 15 and 25 percent. Respondents are asked if they have taken a series of specific actions to reduce energy use in the past 12 months. We group these actions into three major categories: repeated actions such as switching off power strips and turning computers off at night, physical capital changes such as purchasing Energy Star appliances, and intermittent actions such as replacing air filters on air conditioning or heating systems.

Table AI-1 presents the results, combining data across all sites where respondents were asked about an action. Column (1) presents the share of respondents that report taking the action in the past 12 months. For many of the physical capital changes and intermittent actions, the means are too high. While our focus is on the differences between treatment and control, not the means, this does generate concern about whether the surveys yield meaningful responses.

Column (2) shows that there is little difference between treatment and control for the vast majority of actions, and the standard errors are tight enough to detect differences of two to four percentage points. There are three differences: treated households are more likely to use fans to keep cool, have a home energy audit, and participate in utility energy efficiency programs. The latter two actions involve physical capital stock changes. Audits, which are typically offered as part of the utility's energy conservation programs, often include direct installation of new compact fluorescent lightbulbs and can be gateways to other utility programs. Other utility programs often feature subsidies for energy efficient physical capital such as appliances, heating and cooling systems, and insulation. Fortunately, these are the two areas where additional administrative data are available, and we analyze these administrative data in the body of the paper.

For each of the three major categories, the first row (in bold) presents a test of whether the average probability of taking all actions in that category differs between treatment and control. This aggregation across actions gives standard errors tight enough to detect differences of one to two percentage points, but treated households are still not different in any of these three tests. Throughout Table AI- 1 , the failure to reject equality between treatment and control would only be further reinforced by adjusting the p-values for multiple hypothesis testing.

There are multiple interpretations of these results. First, the intervention might increase the true probabilities of taking actions, but the surveys might not pick this up if demand effects, over-reporting, nonresponse, or some other factor differed systematically between treatment and control. However, while the survey results should be interpreted cautiously, it is not obvious what would cause the treatment group to systematically report that they do not take actions. Second, the treatment could cause small changes in the true probabilities of taking a wide variety of actions, none of which are statistically detectable. Such changes could potentially add up to the observed effects on electricity use even though no one action accounts for much on its own. Third, it is possible that the intervention does not affect the "extensive margin" reported in Table AI-1, which is whether or not people take a given action, but instead changes the intensity with which people take actions they were already taking. In other words, an important impact of the intervention could to increase attention and motivation to conserve in the same ways that people were already conserving, instead of giving information about new ways to conserve. 
Table AI-1: Self-Reported Actions

\begin{tabular}{|c|c|c|}
\hline & $(1)$ & $(2)$ \\
\hline "In the past twelve months, have you..." & Mean & Treatment-Control \\
\hline $\begin{array}{l}\text { Taken any steps to } \\
\text { reduce energy use? }\end{array}$ & 0.77 & $\begin{array}{l}0.010 \\
(0.012)\end{array}$ \\
\hline Repeated Actions & 0.62 & $\begin{array}{l}0.005 \\
(0.008)\end{array}$ \\
\hline Adjusted your thermostat settings? & 0.63 & $\begin{array}{l}0.012 \\
(0.015)\end{array}$ \\
\hline Unplugged devices and chargers? & 0.65 & $\begin{array}{l}-0.020 \\
(0.039)\end{array}$ \\
\hline $\begin{array}{l}\text { Switched off power strips } \\
\text { or appliances when unused? }\end{array}$ & 0.59 & $\begin{array}{l}0.002 \\
(0.014)\end{array}$ \\
\hline Turned off lights when unused? & 0.96 & $\begin{array}{c}0.005 \\
(0.009)\end{array}$ \\
\hline Hung laundry to dry? & 0.42 & $\begin{array}{c}0.010 \\
(0.024)\end{array}$ \\
\hline $\begin{array}{l}\text { Used energy saving or sleep } \\
\text { features on your computer? }\end{array}$ & 0.56 & $\begin{array}{l}0.008 \\
(0.021)\end{array}$ \\
\hline Turned off computer at night? & 0.65 & $\begin{array}{l}-0.034 \\
(0.023)\end{array}$ \\
\hline Used fans to keep cool? & 0.80 & $\begin{array}{c}0.072 \\
(0.034) * * \\
\end{array}$ \\
\hline Physical Capital Changes & 0.55 & $\begin{array}{l}-0.002 \\
(0.008)\end{array}$ \\
\hline $\begin{array}{l}\text { Replaced incandescent light bulbs } \\
\text { with LEDs? }\end{array}$ & 0.70 & $\begin{array}{l}0.013 \\
(0.038)\end{array}$ \\
\hline Purchased Energy Star appliances? & 0.74 & $\begin{array}{l}0.002 \\
(0.016)\end{array}$ \\
\hline $\begin{array}{l}\text { Disposed of a second refrigerator } \\
\text { or freezer? }\end{array}$ & 0.26 & $\begin{array}{l}-0.001 \\
(0.015)\end{array}$ \\
\hline Installed light timers or sensors? & 0.30 & $\begin{array}{l}-0.018 \\
(0.038)\end{array}$ \\
\hline $\begin{array}{l}\text { Replaced incandescent light bulbs } \\
\text { with CFLs? }\end{array}$ & 0.81 & $\begin{array}{l}0.000 \\
(0.013)\end{array}$ \\
\hline $\begin{array}{l}\text { Added insulation or } \\
\text { replaced windows? }\end{array}$ & 0.54 & $\begin{array}{l}-0.039 \\
(0.024)\end{array}$ \\
\hline Had a home energy audit? & 0.19 & $\begin{array}{c}0.057 \\
(0.022) * * *\end{array}$ \\
\hline $\begin{array}{l}\text { Installed a programmable } \\
\text { thermostat? }\end{array}$ & 0.79 & $\begin{array}{l}-0.033 \\
(0.032)\end{array}$ \\
\hline$\overline{\text { Intermittent Actions }}$ & 0.62 & $\begin{array}{l}0.006 \\
(0.012)\end{array}$ \\
\hline Tuned up your AC system? & 0.63 & $\begin{array}{l}-0.016 \\
(0.018)\end{array}$ \\
\hline Used a programmable thermostat? & 0.59 & $\begin{array}{l}0.009 \\
(0.028)\end{array}$ \\
\hline $\begin{array}{l}\text { Added weather-stripping or } \\
\text { caulking around windows? }\end{array}$ & 0.60 & $\begin{array}{l}0.008 \\
(0.018)\end{array}$ \\
\hline $\begin{array}{l}\text { Cleaned or replaced heating or } \\
\text { AC system air filters? }\end{array}$ & 0.70 & $\begin{array}{l}0.017 \\
(0.038)\end{array}$ \\
\hline $\begin{array}{l}\text { Participated in any utility } \\
\text { energy effiency programs? }\end{array}$ & 0.19 & $\begin{array}{l}0.018 \\
(0.010) *\end{array}$ \\
\hline Total number of surveys & 5856 & \\
\hline
\end{tabular}


Notes: This table presents survey data on self-reported energy conservation actions. Robust standard errors. ${ }^{*}, * *, * * *$ : Statistically significant with 90,95 , and 99 percent confidence, respectively. 


\section{Appendix Tables}

\section{Notes for Tables A1-A4}

Tables A1-A4 present alternative estimates of Tables 2 and 3. Tables A1 and A2 present alternative estimates of Equation (??) for the first four reports and all later reports, respectively. Tables A3 and A4 similarly present alternative estimates of Equation (??) for the first four reports and all later reports. All tables include the bimonthly group as well as the monthly and quarterly groups.

Within each table, there are two panels. In the first, the left column excludes weather controls, while the right column exactly replicates the estimates in the body of the paper, also reporting the estimated weather coefficients. In the second, the left column excludes outliers: all observations of $Y_{i t}$ greater than $300 \mathrm{kWh} /$ day and all households $i$ with average baseline usage greater than $150 \mathrm{kWh} /$ day, which is five times the mean. Based on our inspection of the data, these high-usage observations appear to be correct, not measurement

errors. However, they implicitly receive significant weight in the OLS estimation, so a small number of high-usage households could in theory drive the results. The right column replaces the original $\mathbf{M}_{i t}$ with six variables: $1\left(C D D_{i t}\right)>0, C D D_{i t}, 1\left(0<H D D_{i t} \leq 5\right), 1\left(5<H D D_{i t} \leq 35\right), H D D_{i t} \cdot 1\left(5<H D D_{i t} \leq 35\right)$, and $1\left(H D D_{i t}>35\right)$. This function was based on inspection of the relationship between ATEs and degree days for this site.

The outcome variable is electricity use, in kilowatt-hours per day. Standard errors are robust, clustered by household. *, **, ***: Statistically significant with 90,95 , and 99 percent confidence, respectively. 
Table A1: Robustness Checks for Table 2, First Four Reports

\begin{tabular}{|c|c|c|c|c|c|c|}
\hline & (1) & $(2)$ & $(3)$ & $(4)$ & $(5)$ & $(6)$ \\
\hline & $\begin{array}{l}\text { Monthly } \\
\text { Base }\end{array}$ & $\begin{array}{l}\text { Monthly } \\
\text { Weather }\end{array}$ & $\begin{array}{c}\text { Quarterly } \\
\text { Base }\end{array}$ & $\begin{array}{l}\text { Quarterly } \\
\text { Weather }\end{array}$ & $\begin{array}{c}\text { Bimonthly } \\
\text { Base }\end{array}$ & $\begin{array}{c}\text { Bimonthly } \\
\text { Weather }\end{array}$ \\
\hline 1 (Treated) $\cdot 1$ (Post-Arrival Period) & $\begin{array}{c}-0.172 \\
(0.030)^{* * *}\end{array}$ & $\begin{array}{c}-0.185 \\
(0.027)^{* * *}\end{array}$ & $\begin{array}{c}-0.201 \\
(0.041)^{* * *}\end{array}$ & $\begin{array}{c}-0.197 \\
(0.035)^{* * *}\end{array}$ & $\begin{array}{c}-0.129 \\
(0.038)^{* * *}\end{array}$ & $\begin{array}{c}-0.152 \\
(0.036)^{* * *}\end{array}$ \\
\hline 1 (Treated) $\cdot 1$ (Arrival Period) & $\begin{array}{c}-0.062 \\
(0.024)^{* * *}\end{array}$ & $\begin{array}{c}-0.062 \\
(0.024)^{* * *}\end{array}$ & $\begin{array}{c}-0.067 \\
(0.029)^{* *}\end{array}$ & $\begin{array}{c}-0.070 \\
(0.028)^{* *}\end{array}$ & $\begin{array}{l}-0.047 \\
(0.033)\end{array}$ & $\begin{array}{l}-0.043 \\
(0.033)\end{array}$ \\
\hline 1 (Treated) & $\begin{array}{c}-0.534 \\
(0.065)^{* * *}\end{array}$ & $\begin{array}{c}-0.451 \\
(0.086)^{* * *}\end{array}$ & $\begin{array}{c}-0.391 \\
(0.067)^{* * *}\end{array}$ & $\begin{array}{c}-0.420 \\
(0.084)^{* * *}\end{array}$ & $\begin{array}{c}-0.366 \\
(0.059)^{* * *}\end{array}$ & $\begin{array}{c}-0.276 \\
(0.106)^{* * *}\end{array}$ \\
\hline 1 (Treated) $\cdot$ Heating Degrees & & $\begin{array}{l}-0.004 \\
(0.004)\end{array}$ & & $\begin{array}{c}0.002 \\
(0.005)\end{array}$ & & $\begin{array}{l}-0.009 \\
(0.008)\end{array}$ \\
\hline Heating Degrees & & $\begin{array}{c}0.038 \\
(0.016)^{* *}\end{array}$ & & $\begin{array}{c}0.020 \\
(0.014)\end{array}$ & & $\begin{array}{c}0.083 \\
(0.011)^{* * *}\end{array}$ \\
\hline 1 (Treated) $\cdot$ Cooling Degrees & & & & $\begin{array}{c}0.000 \\
(0.010)\end{array}$ & & $\begin{array}{c}-0.031 \\
(0.019)^{*}\end{array}$ \\
\hline Cooling Degrees & & & & $\begin{array}{c}0.281 \\
(0.019)^{* * *}\end{array}$ & & $\begin{array}{c}0.016 \\
(0.027)\end{array}$ \\
\hline$N$ & $8,515,691$ & $8,515,691$ & $19,333,058$ & $19,333,058$ & $9,609,303$ & $9,609,303$ \\
\hline
\end{tabular}

\begin{tabular}{|c|c|c|c|c|c|c|}
\hline & $(1)$ & $(2)$ & $(3)$ & $(4)$ & $(5)$ & (6) \\
\hline & $\begin{array}{l}\text { Monthly } \\
\text { Outliers }\end{array}$ & $\begin{array}{c}\text { Monthly } \\
\text { Full M }\end{array}$ & $\begin{array}{l}\text { Quarterly } \\
\text { Outliers }\end{array}$ & $\begin{array}{c}\text { Quarterly } \\
\text { Full M }\end{array}$ & $\begin{array}{l}\text { Bimonthly } \\
\text { Outliers }\end{array}$ & $\begin{array}{c}\text { Bimonthly } \\
\text { Full M }\end{array}$ \\
\hline 1 (Treated) $\cdot 1($ Post-Arrival Period $)$ & $\begin{array}{c}-0.183 \\
(0.027)^{* * *}\end{array}$ & $\begin{array}{c}-0.185 \\
(0.027)^{* * *}\end{array}$ & $\begin{array}{c}-0.190 \\
(0.036)^{* * *}\end{array}$ & $\begin{array}{c}-0.193 \\
(0.035)^{* * *}\end{array}$ & $\begin{array}{c}-0.154 \\
(0.034)^{* * *}\end{array}$ & $\begin{array}{c}-0.160 \\
(0.036)^{* * *}\end{array}$ \\
\hline 1 (Treated) $\cdot 1$ (Arrival Period) & $\begin{array}{c}-0.061 \\
(0.024)^{* *}\end{array}$ & $\begin{array}{c}-0.061 \\
(0.023)^{* * *}\end{array}$ & $\begin{array}{c}-0.070 \\
(0.028)^{* *}\end{array}$ & $\begin{array}{c}-0.069 \\
(0.028)^{* *}\end{array}$ & $\begin{array}{l}-0.032 \\
(0.031)\end{array}$ & $\begin{array}{c}-0.052 \\
(0.029)^{*}\end{array}$ \\
\hline 1 (Treated) & $\begin{array}{c}-0.430 \\
(0.086)^{* * *}\end{array}$ & $\begin{array}{c}-0.580 \\
(0.114)^{* * *}\end{array}$ & $\begin{array}{c}-0.413 \\
(0.083)^{* * *}\end{array}$ & $\begin{array}{c}-0.408 \\
(0.091)^{* * *}\end{array}$ & $\begin{array}{c}-0.228 \\
(0.101)^{* *}\end{array}$ & $\begin{array}{c}-0.379 \\
(0.103)^{* * *}\end{array}$ \\
\hline 1 (Treated) $\cdot$ Heating Degrees & $\begin{array}{l}-0.004 \\
(0.004)\end{array}$ & & $\begin{array}{c}0.002 \\
(0.005)\end{array}$ & & $\begin{array}{l}-0.011 \\
(0.007)\end{array}$ & \\
\hline Heating Degrees & $\begin{array}{c}0.039 \\
(0.016)^{* *}\end{array}$ & & $\begin{array}{c}0.021 \\
(0.014)\end{array}$ & & $\begin{array}{c}0.089 \\
(0.010)^{* * *}\end{array}$ & \\
\hline 1 (Treated) $\cdot$ Cooling Degrees & & & $\begin{array}{c}0.000 \\
(0.010)\end{array}$ & & $\begin{array}{l}-0.018 \\
(0.016)\end{array}$ & \\
\hline Cooling Degrees & & & $\begin{array}{c}0.279 \\
(0.019)^{* * *}\end{array}$ & & $\begin{array}{l}-0.016 \\
(0.022)\end{array}$ & \\
\hline$N$ & $8,514,078$ & $8,515,691$ & $19,330,176$ & $19,333,058$ & $9,589,391$ & $9,609,303$ \\
\hline
\end{tabular}


Table A2: Robustness Checks for Table 2, Later Reports

\begin{tabular}{|c|c|c|c|c|c|c|}
\hline & $(1)$ & $(2)$ & (3) & (4) & $(5)$ & (6) \\
\hline & $\begin{array}{l}\text { Monthly } \\
\text { Base }\end{array}$ & $\begin{array}{l}\text { Monthly } \\
\text { Weather }\end{array}$ & $\begin{array}{c}\text { Quarterly } \\
\text { Base }\end{array}$ & $\begin{array}{l}\text { Quarterly } \\
\text { Weather }\end{array}$ & $\begin{array}{c}\text { Bimonthly } \\
\text { Base }\end{array}$ & $\begin{array}{c}\text { Bimonthly } \\
\text { Weather }\end{array}$ \\
\hline 1 (Treated) $\cdot 1$ (Post-Arrival Period) & $\begin{array}{c}-0.032 \\
(0.009)^{* * *}\end{array}$ & $\begin{array}{c}-0.033 \\
(0.009)^{* * *}\end{array}$ & $\begin{array}{c}-0.045 \\
(0.022)^{* *}\end{array}$ & $\begin{array}{c}-0.038 \\
(0.022)^{*}\end{array}$ & $\begin{array}{c}-0.211 \\
(0.060)^{* * *}\end{array}$ & $\begin{array}{c}-0.230 \\
(0.061)^{* * *}\end{array}$ \\
\hline 1 (Treated) $\cdot 1$ (Arrival Period) & $\begin{array}{c}-0.015 \\
(0.007)^{* *}\end{array}$ & $\begin{array}{c}-0.017 \\
(0.007)^{* *}\end{array}$ & $\begin{array}{l}-0.010 \\
(0.019)\end{array}$ & $\begin{array}{l}-0.005 \\
(0.019)\end{array}$ & $\begin{array}{l}-0.025 \\
(0.047)\end{array}$ & $\begin{array}{c}-0.129 \\
(0.049)^{* * *}\end{array}$ \\
\hline 1(Treated) & $\begin{array}{c}-0.801 \\
(0.058) * * *\end{array}$ & $\begin{array}{c}-0.706 \\
(0.059)^{* * *}\end{array}$ & $\begin{array}{c}-0.657 \\
(0.092)^{* * *}\end{array}$ & $\begin{array}{c}-0.509 \\
(0.095)^{* * *}\end{array}$ & $\begin{array}{c}-0.645 \\
(0.089)^{* * *}\end{array}$ & $\begin{array}{l}-0.048 \\
(0.143)\end{array}$ \\
\hline 1 (Treated) $\cdot$ Heating Degrees & & $\begin{array}{c}-0.006 \\
(0.003)^{* *}\end{array}$ & & $\begin{array}{c}-0.010 \\
(0.005)^{* *}\end{array}$ & & $\begin{array}{c}-0.034 \\
(0.008)^{* * *}\end{array}$ \\
\hline 1 (Treated) $\cdot$ Cooling Degrees & & $\begin{array}{c}-0.017 \\
(0.007)^{* *}\end{array}$ & & $\begin{array}{l}-0.007 \\
(0.013)\end{array}$ & & $\begin{array}{c}-0.050 \\
(0.028)^{*}\end{array}$ \\
\hline Heating Degrees & & $\begin{array}{c}0.004 \\
(0.010)\end{array}$ & & $\begin{array}{c}0.007 \\
(0.011)\end{array}$ & & $\begin{array}{c}0.082 \\
(0.013)^{* * *}\end{array}$ \\
\hline Cooling Degrees & & $\begin{array}{c}0.090 \\
(0.012)^{* * *}\end{array}$ & & $\begin{array}{c}0.023 \\
(0.015)\end{array}$ & & $\begin{array}{c}0.463 \\
(0.029)^{* * *}\end{array}$ \\
\hline$N$ & $75,217,587$ & $75,217,587$ & $52,418,516$ & $52,418,516$ & $19,554,914$ & $19,554,914$ \\
\hline
\end{tabular}

\begin{tabular}{|c|c|c|c|c|c|c|}
\hline & (1) & (2) & (3) & $(4)$ & $(5)$ & (6) \\
\hline & $\begin{array}{l}\text { Monthly } \\
\text { Outliers }\end{array}$ & $\begin{array}{c}\text { Monthly } \\
\text { Full M }\end{array}$ & $\begin{array}{c}\text { Quarterly } \\
\text { Outliers }\end{array}$ & $\begin{array}{l}\text { Quarterly } \\
\text { Full M }\end{array}$ & $\begin{array}{c}\text { Bimonthly } \\
\text { Outliers }\end{array}$ & $\begin{array}{c}\text { Bimonthly } \\
\text { Full M }\end{array}$ \\
\hline 1 (Treated) $\cdot 1$ (Post-Arrival Period) & $\begin{array}{c}-0.030 \\
(0.009)^{* * *}\end{array}$ & $\begin{array}{c}-0.032 \\
(0.009)^{* * *}\end{array}$ & $\begin{array}{l}-0.036 \\
(0.022)\end{array}$ & $\begin{array}{l}-0.035 \\
(0.022)\end{array}$ & $\begin{array}{c}-0.217 \\
(0.060)^{* * *}\end{array}$ & $\begin{array}{c}-0.233 \\
(0.061)^{* * *}\end{array}$ \\
\hline 1 (Treated) $\cdot 1$ (Arrival Period $)$ & $\begin{array}{c}-0.016 \\
(0.007)^{* *}\end{array}$ & $\begin{array}{c}-0.017 \\
(0.007)^{* *}\end{array}$ & $\begin{array}{l}-0.004 \\
(0.019)\end{array}$ & $\begin{array}{l}-0.006 \\
(0.019)\end{array}$ & $\begin{array}{c}-0.129 \\
(0.049)^{* * *}\end{array}$ & $\begin{array}{c}-0.137 \\
(0.050)^{* * *}\end{array}$ \\
\hline 1(Treated) & $\begin{array}{c}-0.696 \\
(0.059)^{* * *}\end{array}$ & $\begin{array}{c}-0.762 \\
(0.063)^{* * *}\end{array}$ & $\begin{array}{c}-0.509 \\
(0.094)^{* * *}\end{array}$ & $\begin{array}{c}-0.555 \\
(0.115)^{* * *}\end{array}$ & $\begin{array}{l}-0.041 \\
(0.138)\end{array}$ & $\begin{array}{l}-0.144 \\
(0.141)\end{array}$ \\
\hline 1 (Treated) $\cdot$ Heating Degrees & $\begin{array}{c}-0.007 \\
(0.003)^{* *}\end{array}$ & & $\begin{array}{c}-0.009 \\
(0.005)^{*}\end{array}$ & & $\begin{array}{c}-0.034 \\
(0.008)^{* * *}\end{array}$ & \\
\hline 1(Treated) $\cdot$ Cooling Degrees & $\begin{array}{c}-0.017 \\
(0.007)^{* *}\end{array}$ & & $\begin{array}{l}-0.007 \\
(0.013)\end{array}$ & & $\begin{array}{l}-0.042 \\
(0.026)\end{array}$ & \\
\hline Heating Degrees & $\begin{array}{c}0.004 \\
(0.010)\end{array}$ & & $\begin{array}{c}0.007 \\
(0.011)\end{array}$ & & $\begin{array}{c}0.082 \\
(0.012)^{* * *}\end{array}$ & \\
\hline Cooling Degrees & $\begin{array}{c}0.089 \\
(0.012)^{* * *}\end{array}$ & & $\begin{array}{c}0.022 \\
(0.015)\end{array}$ & & $\begin{array}{c}0.444 \\
(0.028)^{* * *}\end{array}$ & \\
\hline$N$ & $75,201,504$ & $75,217,587$ & $52,409,856$ & $52,418,516$ & $19,513,453$ & $19,554,914$ \\
\hline
\end{tabular}


Table A3: Robustness Checks for Table 3, First Four Reports

\begin{tabular}{|c|c|c|c|c|c|c|}
\hline & (1) & (2) & (3) & (4) & (5) & $(6)$ \\
\hline & $\begin{array}{l}\text { Monthly } \\
\text { Base }\end{array}$ & $\begin{array}{l}\text { Monthly } \\
\text { Weather }\end{array}$ & $\begin{array}{c}\text { Quarterly } \\
\text { Base }\end{array}$ & $\begin{array}{l}\text { Quarterly } \\
\text { Weather }\end{array}$ & $\begin{array}{c}\text { Bimonthly } \\
\text { Base }\end{array}$ & $\begin{array}{l}\text { Bimonthly } \\
\text { Weather }\end{array}$ \\
\hline 1 (Treated) $\cdot 1$ (Window) $\cdot$ Time & $\begin{array}{l}1.356 \\
(1.265)\end{array}$ & $\begin{array}{c}4.082 \\
(1.302)^{* * *}\end{array}$ & $\begin{array}{c}0.706 \\
(0.195)^{* * *}\end{array}$ & $\begin{array}{c}0.708 \\
(0.187)^{* * *}\end{array}$ & $\begin{array}{c}1.012 \\
(0.439)^{* *}\end{array}$ & $\begin{array}{c}0.948 \\
(0.426)^{* *}\end{array}$ \\
\hline 1(Treated) & $\begin{array}{c}-0.413 \\
(0.064)^{* * *}\end{array}$ & $\begin{array}{l}-0.098 \\
(0.095)\end{array}$ & $\begin{array}{c}-0.346 \\
(0.071)^{* * *}\end{array}$ & $\begin{array}{c}-0.338 \\
(0.084)^{* * *}\end{array}$ & $\begin{array}{c}-0.408 \\
(0.067)^{* * *}\end{array}$ & $\begin{array}{c}-0.242 \\
(0.104)^{* *}\end{array}$ \\
\hline 1 (Treated) $\cdot$ Heating Degrees & & $\begin{array}{c}-0.013 \\
(0.004)^{* * *}\end{array}$ & & $\begin{array}{l}-0.000 \\
(0.004)\end{array}$ & & $\begin{array}{l}-0.011 \\
(0.008)\end{array}$ \\
\hline Heating Degrees & & $\begin{array}{c}0.042 \\
(0.016)^{* * *}\end{array}$ & & $\begin{array}{c}0.021 \\
(0.014)\end{array}$ & & $\begin{array}{c}0.085 \\
(0.011)^{* * *}\end{array}$ \\
\hline 1 (Treated) $\cdot$ Cooling Degrees & & & & $\begin{array}{l}-0.004 \\
(0.012)\end{array}$ & & $\begin{array}{l}-0.028 \\
(0.019)\end{array}$ \\
\hline Cooling Degrees & & & & $\begin{array}{c}0.282 \\
(0.019)^{* * *}\end{array}$ & & $\begin{array}{c}0.015 \\
(0.027)\end{array}$ \\
\hline$N$ & $8,515,691$ & $8,515,691$ & $19,333,058$ & $19,333,058$ & $9,609,303$ & $9,609,303$ \\
\hline
\end{tabular}

\begin{tabular}{|c|c|c|c|c|c|c|}
\hline & (1) & (2) & (3) & (4) & (5) & (6) \\
\hline & $\begin{array}{l}\text { Monthly } \\
\text { Outliers }\end{array}$ & $\begin{array}{l}\text { Monthly } \\
\text { Full M }\end{array}$ & $\begin{array}{l}\text { Quarterly } \\
\text { Outliers }\end{array}$ & $\begin{array}{l}\text { Quarterly } \\
\text { Full M }\end{array}$ & $\begin{array}{l}\text { Bimonthly } \\
\text { Outliers }\end{array}$ & $\begin{array}{l}\text { Bimonthly } \\
\text { Full M }\end{array}$ \\
\hline 1 (Treated) $\cdot 1($ Window $) \cdot$ Time & $\begin{array}{c}4.061 \\
(1.290)^{* * *}\end{array}$ & $\begin{array}{c}4.476 \\
(1.309)^{* * *}\end{array}$ & $\begin{array}{c}0.674 \\
(0.185)^{* * *}\end{array}$ & $\begin{array}{c}0.697 \\
(0.187)^{* * *}\end{array}$ & $\begin{array}{c}0.744 \\
(0.392)^{*}\end{array}$ & $\begin{array}{c}0.884 \\
(0.417)^{* *}\end{array}$ \\
\hline 1(Treated) & $\begin{array}{l}-0.083 \\
(0.094)\end{array}$ & $\begin{array}{c}-0.544 \\
(0.120)^{* * *}\end{array}$ & $\begin{array}{c}-0.325 \\
(0.083)^{* * *}\end{array}$ & $\begin{array}{c}-0.319 \\
(0.089)^{* * *}\end{array}$ & $\begin{array}{c}-0.222 \\
(0.101)^{* *}\end{array}$ & $\begin{array}{c}-0.342 \\
(0.101)^{* * *}\end{array}$ \\
\hline 1 (Treated) $\cdot$ Heating Degrees & $\begin{array}{c}-0.014 \\
(0.004)^{* * *}\end{array}$ & & $\begin{array}{l}-0.001 \\
(0.004)\end{array}$ & & $\begin{array}{l}-0.011 \\
(0.007)\end{array}$ & \\
\hline Heating Degrees & $\begin{array}{c}0.043 \\
(0.016)^{* * *}\end{array}$ & & $\begin{array}{c}0.021 \\
(0.014)\end{array}$ & & $\begin{array}{c}0.089 \\
(0.010)^{* * *}\end{array}$ & \\
\hline 1 (Treated) $\cdot$ Cooling Degrees & & & $\begin{array}{l}-0.004 \\
(0.012)\end{array}$ & & $\begin{array}{l}-0.010 \\
(0.016)\end{array}$ & \\
\hline Cooling Degrees & & & $\begin{array}{c}0.280 \\
(0.019)^{* * *}\end{array}$ & & $\begin{array}{l}-0.020 \\
(0.022)\end{array}$ & \\
\hline$N$ & $8,514,078$ & $8,515,691$ & $19,330,176$ & $19,333,058$ & $9,589,391$ & $9,609,303$ \\
\hline
\end{tabular}


Table A4: Robustness Checks for Table 3, After First Four Reports

\begin{tabular}{|c|c|c|c|c|c|c|}
\hline & $(1)$ & $(2)$ & (3) & $(4)$ & $(5)$ & (6) \\
\hline & $\begin{array}{c}\text { Monthly } \\
\text { Base }\end{array}$ & $\begin{array}{l}\text { Monthly } \\
\text { Weather }\end{array}$ & $\begin{array}{c}\text { Quarterly } \\
\text { Base }\end{array}$ & $\begin{array}{l}\text { Quarterly } \\
\text { Weather }\end{array}$ & $\begin{array}{c}\text { Bimonthly } \\
\text { Base }\end{array}$ & $\begin{array}{c}\text { Bimonthly } \\
\text { Weather }\end{array}$ \\
\hline 1 (Treated) $\cdot 1$ (Window) $\cdot$ Time & $\begin{array}{c}0.333 \\
(0.322)\end{array}$ & $\begin{array}{c}0.393 \\
(0.315)\end{array}$ & $\begin{array}{c}0.017 \\
(0.141)\end{array}$ & $\begin{array}{c}0.023 \\
(0.140)\end{array}$ & $\begin{array}{c}0.022 \\
(0.539)\end{array}$ & $\begin{array}{c}0.134 \\
(0.536)\end{array}$ \\
\hline 1(Treated) & $\begin{array}{c}-0.777 \\
(0.056) * * *\end{array}$ & $\begin{array}{c}-0.682 \\
(0.058)^{* * *}\end{array}$ & $\begin{array}{c}-0.606 \\
(0.087)^{* * *}\end{array}$ & $\begin{array}{c}-0.532 \\
(0.091)^{* * *}\end{array}$ & $\begin{array}{c}-0.551 \\
(0.089)^{* * *}\end{array}$ & $\begin{array}{l}-0.080 \\
(0.141)\end{array}$ \\
\hline 1(Treated) $\cdot$ Heating Degrees & & $\begin{array}{c}-0.007 \\
(0.003)^{* *}\end{array}$ & & $\begin{array}{l}-0.006 \\
(0.004)\end{array}$ & & $\begin{array}{c}-0.030 \\
(0.008)^{* * *}\end{array}$ \\
\hline 1 (Treated) $\cdot$ Cooling Degrees & & $\begin{array}{c}-0.014 \\
(0.008)^{*}\end{array}$ & & $\begin{array}{l}-0.005 \\
(0.013)\end{array}$ & & $\begin{array}{l}-0.044 \\
(0.029)\end{array}$ \\
\hline Heating Degrees & & $\begin{array}{c}0.004 \\
(0.010)\end{array}$ & & $\begin{array}{c}0.007 \\
(0.011)\end{array}$ & & $\begin{array}{c}0.080 \\
(0.013)^{* * *}\end{array}$ \\
\hline Cooling Degrees & & $\begin{array}{c}0.089 \\
(0.012)^{* * *}\end{array}$ & & $\begin{array}{c}0.023 \\
(0.015)\end{array}$ & & $\begin{array}{c}0.460 \\
(0.029)^{* * *}\end{array}$ \\
\hline$N$ & $75,217,587$ & $75,217,587$ & $52,418,516$ & $52,418,516$ & $19,554,914$ & $19,554,914$ \\
\hline
\end{tabular}

\begin{tabular}{|c|c|c|c|c|c|c|}
\hline & (1) & (2) & (3) & $(4)$ & (5) & $(6)$ \\
\hline & $\begin{array}{l}\text { Monthly } \\
\text { Outliers }\end{array}$ & $\begin{array}{l}\text { Monthly } \\
\text { Full M }\end{array}$ & $\begin{array}{l}\text { Quarterly } \\
\text { Outliers }\end{array}$ & $\begin{array}{l}\text { Quarterly } \\
\text { Full M }\end{array}$ & $\begin{array}{c}\text { Bimonthly } \\
\text { Outliers }\end{array}$ & $\begin{array}{l}\text { Bimonthly } \\
\text { Full M }\end{array}$ \\
\hline 1 (Treated) $\cdot 1$ (Window) $\cdot$ Time & $\begin{array}{c}0.312 \\
(0.313)\end{array}$ & $\begin{array}{c}0.449 \\
(0.312)\end{array}$ & $\begin{array}{c}0.037 \\
(0.140)\end{array}$ & $\begin{array}{c}0.023 \\
(0.141)\end{array}$ & $\begin{array}{l}-0.115 \\
(0.526)\end{array}$ & $\begin{array}{c}0.275 \\
(0.528)\end{array}$ \\
\hline 1(Treated) & $\begin{array}{c}-0.672 \\
(0.058)^{* * *}\end{array}$ & $\begin{array}{c}-0.725 \\
(0.061)^{* * *}\end{array}$ & $\begin{array}{c}-0.532 \\
(0.091)^{* * *}\end{array}$ & $\begin{array}{c}-0.509 \\
(0.104)^{* * *}\end{array}$ & $\begin{array}{l}-0.070 \\
(0.136)\end{array}$ & $\begin{array}{l}-0.144 \\
(0.141)\end{array}$ \\
\hline 1 (Treated) $\cdot$ Heating Degrees & $\begin{array}{c}-0.007 \\
(0.003)^{* *}\end{array}$ & & $\begin{array}{l}-0.005 \\
(0.004)\end{array}$ & & $\begin{array}{c}-0.031 \\
(0.008)^{* * *}\end{array}$ & \\
\hline 1 (Treated) $\cdot$ Cooling Degrees & $\begin{array}{c}-0.014 \\
(0.008)^{*}\end{array}$ & & $\begin{array}{l}-0.004 \\
(0.013)\end{array}$ & & $\begin{array}{l}-0.037 \\
(0.026)\end{array}$ & \\
\hline Heating Degrees & $\begin{array}{c}0.004 \\
(0.010)\end{array}$ & & $\begin{array}{c}0.007 \\
(0.011)\end{array}$ & & $\begin{array}{c}0.080 \\
(0.012)^{* * *}\end{array}$ & \\
\hline Cooling Degrees & $\begin{array}{c}0.088 \\
(0.012)^{* * *}\end{array}$ & & $\begin{array}{c}0.021 \\
(0.015)\end{array}$ & & $\begin{array}{c}0.441 \\
(0.028)^{* * *}\end{array}$ & \\
\hline$N$ & $75,201,504$ & $75,217,587$ & $52,409,856$ & $52,418,516$ & $19,513,453$ & $19,554,914$ \\
\hline
\end{tabular}




\section{Table A5: Placebo Report Arrivals}

\begin{tabular}{lcc}
\hline & $(1)$ & $(2)$ \\
\hline & Base & Weather \\
\hline 1 (Treated) $\cdot 1$ (Arrival Period) & -0.001 & -0.007 \\
& $(0.016)$ & $(0.015)$ \\
1 (Treated) $\cdot 1$ (Post-Arrival Period) & 0.011 & -0.004 \\
& $(0.019)$ & $(0.019)$ \\
1 (Treated) & -0.671 & -0.489 \\
& $(0.095)^{* * *}$ & $(0.093)^{* * *}$ \\
1 (Treated) $\cdot$ Heating Degrees & & -0.012 \\
& & $(0.005)^{* *}$ \\
1 (Treated) $\cdot$ Cooling Degrees & & -0.008 \\
& & $(0.013)$ \\
Heating Degrees & & 0.007 \\
& & $(0.011)$ \\
Cooling Degrees & & 0.023 \\
$N$ & & $(0.015)$ \\
& $52,418,516$ & $52,418,516$ \\
\hline
\end{tabular}

Notes: This table presents the estimates of Equation (??) for the quarterly group, for reports that the monthly group received but the quarterly group did not. The sample includes the period after the quarterly group's first four reports. The left column does not control for degree days, while the right column does. The outcome variable is electricity use, in kilowatt-hours per day. Standard errors are robust, clustered by household. *, **, ***: Statistically significant with 90,95 , and 99 percent confidence, respectively. 
Table A6: Persistence by Subgroup

\begin{tabular}{|c|c|c|c|c|c|c|}
\hline & (1) & $(2)$ & (3) & (4) & $(5)$ & (6) \\
\hline & $\begin{array}{l}\text { Site } 1 \\
\text { Levels }\end{array}$ & $\begin{array}{c}\text { Site } 1 \\
\text { Decays }\end{array}$ & $\begin{array}{l}\text { Site } 2 \\
\text { Levels }\end{array}$ & $\begin{array}{c}\text { Site } 2 \\
\text { Decays }\end{array}$ & $\begin{array}{c}\text { Both Sites } \\
\text { Levels }\end{array}$ & $\begin{array}{c}\text { Both Sites } \\
\text { Decavs }\end{array}$ \\
\hline 1(Dropped) & $\begin{array}{c}-0.601 \\
(0.090)^{* * *}\end{array}$ & $\begin{array}{c}-0.832 \\
(0.097)^{* * *}\end{array}$ & $\begin{array}{c}-0.650 \\
(0.101)^{* * *}\end{array}$ & $\begin{array}{c}-0.805 \\
(0.108)^{* * *}\end{array}$ & $\begin{array}{c}-0.626 \\
(0.068)^{* * *}\end{array}$ & $\begin{array}{c}-0.812 \\
(0.072)^{* * *}\end{array}$ \\
\hline 1(Dropped) $\cdot 1$ (Quarterly) & $\begin{array}{c}0.077 \\
(0.188)\end{array}$ & $\begin{array}{c}0.324 \\
(0.202)\end{array}$ & $\begin{array}{c}0.233 \\
(0.177)\end{array}$ & $\begin{array}{c}0.293 \\
(0.190)\end{array}$ & $\begin{array}{c}0.169 \\
(0.131)\end{array}$ & $\begin{array}{c}0.290 \\
(0.139)^{* *}\end{array}$ \\
\hline 1(Dropped) $\cdot$ Baseline Usage & $\begin{array}{c}-0.283 \\
(0.163)^{*}\end{array}$ & $\begin{array}{c}-0.477 \\
(0.184)^{* * *}\end{array}$ & $\begin{array}{c}-0.632 \\
(0.142)^{* * *}\end{array}$ & $\begin{array}{c}-0.561 \\
(0.154)^{* * *}\end{array}$ & $\begin{array}{c}-0.476 \\
(0.107)^{* * *}\end{array}$ & $\begin{array}{c}-0.495 \\
(0.119)^{* * *}\end{array}$ \\
\hline 1 (Dropped) $\cdot 1$ (Post-Drop) x Time & & $\begin{array}{c}0.211 \\
(0.057)^{* * *}\end{array}$ & & $\begin{array}{c}0.131 \\
(0.054)^{* *}\end{array}$ & & $\begin{array}{c}0.164 \\
(0.040)^{* * *}\end{array}$ \\
\hline Quarterly Decay Difference & & $\begin{array}{c}-0.232 \\
(0.122)^{*}\end{array}$ & & $\begin{array}{l}-0.050 \\
(0.093)\end{array}$ & & $\begin{array}{l}-0.109 \\
(0.075)\end{array}$ \\
\hline Baseline Usage Decay Difference & & $\begin{array}{c}0.183 \\
(0.111)^{*}\end{array}$ & & $\begin{array}{l}-0.061 \\
(0.081)\end{array}$ & & $\begin{array}{c}0.017 \\
(0.067)\end{array}$ \\
\hline$N$ & 956,848 & 956,848 & $1,387,473$ & $1,387,473$ & $2,344,321$ & $2,344,321$ \\
\hline
\end{tabular}

Notes: This table presents the estimates of Equation (??), allowing $\gamma$ and $\delta^{L R}$ to differ for monthly vs. quarterly groups and as a function of $\widetilde{Y}^{b}$, which is baseline usage normalized to mean 0 , standard deviation 1. The sample is limited to the post-drop period and includes only dropped and control group households. The outcome variable is monthly average electricity use, in kilowatt-hours per day. Standard errors are robust, clustered by household. *, **, ***: Statistically significant with 90, 95, and 99 percent confidence, respectively. 
Table A7: Table 4 with Balanced Panel

\begin{tabular}{lccc}
\hline & $(1)$ & $(2)$ & $(3)$ \\
\hline & Site 1 & Site 2 & Site 3 \\
\hline 1 (Treated) $\cdot 1$ (Pre-Treatment) & 0.033 & -0.039 & -0.010 \\
& $(0.088)$ & $(0.055)$ & $(0.073)$ \\
1 (Treated) $\cdot 1$ (1st Year) & -0.565 & -0.515 & -0.652 \\
& $(0.065)^{* * *}$ & $(0.065)^{* * *}$ & $(0.093)^{* * *}$ \\
1 (Treated) $\cdot 1$ (2nd Year Until Drop) & -0.882 & -0.701 & -0.859 \\
& $(0.076)^{* * *}$ & $(0.078)^{* * *}$ & $(0.102)^{* * *}$ \\
1 (Treated) $\cdot 1$ (Post-Drop) & -0.605 & -0.554 & -0.618 \\
& $(0.088)^{* * *}$ & $(0.093)^{* * *}$ & $(0.126)^{* * *}$ \\
$1($ Continued) $\cdot 1$ (Pre-Treatment) & -0.045 & 0.023 & -0.038 \\
& $(0.093)$ & $(0.060)$ & $(0.055)$ \\
$1($ Continued) $\cdot 1$ 1 1st Year) & -0.018 & 0.070 & -0.110 \\
& $(0.067)$ & $(0.071)$ & $(0.066)^{*}$ \\
$1($ Continued) $\cdot 1$ (2nd Year Until Drop) & 0.005 & 0.045 & -0.070 \\
& $(0.078)$ & $(0.085)$ & $(0.075)$ \\
$1($ Continued) $\cdot 1$ (Post-Drop) & -0.329 & -0.299 & -0.418 \\
& $(0.089)^{* * *}$ & $(0.100)^{* * *}$ & $(0.095)^{* * *}$ \\
$N$ & $2,924,939$ & $3,800,809$ & $4,226,607$ \\
\hline
\end{tabular}

Notes: This table presents the estimates of Equation (??), omitting the third and fourth lines, with the sample limited to households that never move. It replicates Table 4, except with a balanced panel. The outcome variable is monthly average electricity use, in kilowatt-hours per day. Standard errors are robust and clustered by household in Sites 1 and 2 and by block batch in Site 3. *, **, ***: Statistically significant with 90, 95, and 99 percent confidence, respectively. 
Table A8: Robustness Checks for Table 5

\begin{tabular}{lcccccc}
\hline & $(1)$ & $(2)$ & $(3)$ & $(4)$ & $(5)$ & $(6)$ \\
\hline & Site 1 & Site 1 & Site 2 & Site 2 & Site 3 & Site 3 \\
& No Weather & Balanced & No Weather & Balanced & No Weather & Balanced \\
\hline 1 (Treated) $\cdot 1$ (1st Year) & -0.565 & -0.578 & -0.450 & -0.469 & -0.641 & -0.722 \\
& $(0.042)^{* * *}$ & $(0.045)^{* * *}$ & $(0.043)^{* * *}$ & $(0.045)^{* * *}$ & $(0.084)^{* * *}$ & $(0.083)^{* * *}$ \\
1 (Treated) $\cdot 1$ (2nd Year Until Drop) & -0.867 & -0.923 & -0.659 & -0.597 & -0.865 & -0.783 \\
& $(0.053)^{* * *}$ & $(0.065)^{* * *}$ & $(0.052)^{* * *}$ & $(0.065)^{* * *}$ & $(0.092)^{* * *}$ & $(0.106)^{* * *}$ \\
1 (Treated) $\cdot 1$ (Post-Drop) & -0.786 & -0.826 & -0.718 & -0.595 & -0.725 & -0.551 \\
& $(0.090)^{* * *}$ & $(0.093)^{* * *}$ & $(0.095)^{* * *}$ & $(0.091)^{* * *}$ & $(0.129)^{* * *}$ & $(0.130)^{* * *}$ \\
1 (Continued) $\cdot 1$ (Post-Drop) & -0.243 & -0.188 & -0.087 & -0.183 & -0.163 & -0.422 \\
& $(0.091)^{* * *}$ & $(0.098)^{*}$ & $(0.105)$ & $(0.103)^{*}$ & $(0.106)$ & $(0.108)^{* * *}$ \\
1 (Dropped) $\cdot 1$ (Post-Drop) x Time & 0.176 & 0.161 & 0.114 & 0.106 & 0.086 & 0.080 \\
& $(0.053)^{* * *}$ & $(0.050)^{* * *}$ & $(0.047)^{* *}$ & $(0.044)^{* *}$ & $(0.045)^{*}$ & $(0.044)^{*}$ \\
1 (Continued) $\cdot 1$ (Post-Drop) x Time & 0.091 & 0.067 & -0.062 & -0.067 & -0.079 & -0.044 \\
& $(0.041)^{* *}$ & $(0.039)^{*}$ & $(0.039)$ & $(0.036)^{*}$ & $(0.034)^{* *}$ & $(0.034)$ \\
$N$ & $3,294,294$ & $2,924,939$ & $4,435,689$ & $3,800,809$ & $5,063,949$ & $4,226,607$ \\
Weather Controls & No & Yes & No & Yes & No & Yes \\
\hline
\end{tabular}

Notes: This table presents the estimates of Equation (??), omitting the second line. These are robustness checks for Table 5. Within each site, the left column excludes weather controls, while the right column limits the sample to households that never move. The outcome variable is monthly average electricity use, in kilowatt-hours per day. Standard errors are robust and clustered by household in Sites 1 and 2 and by block batch in Site 3. * **, ***: Statistically significant with 90,95 , and 99 percent confidence, respectively. 
Table A9: Lifetime Extrapolated Cost Effectiveness for the Dropped Group Design

\begin{tabular}{lccc}
\hline Site & 1 & 2 & 3 \\
\hline Program cost (\$/household) & & & \\
\hline & 17 & 18 & 20 \\
Electricity Savings (kWh/household) & & & \\
\hline Savings during treatment & 523 & 405 & 628 \\
$\quad$ Standard Error) & $(25)$ & $(25)$ & $(52)$ \\
Post-treatment savings & 889 & 798 & 1491 \\
$\quad$ Standard Error) & $(54)$ & $(63)$ & $(159)$ \\
Total savings & 1412 & 1203 & 2119 \\
$\quad$ Standard Error) & $(60)$ & $(68)$ & $(167)$ \\
& & & \\
Cost Effectiveness (cents/kWh) & & & \\
\hline Zero Persistence Assumption & 3.31 & 4.44 & \\
$\quad$ Standard Error) & $(0.16)$ & $(0.27)$ & $(0.26)$ \\
Observed Persistence $\quad 1.23$ & 1.49 & 0.95 \\
$\quad$ Standard Error) & $(0.05)$ & $(0.08)$ & $(0.07)$ \\
$\quad$ & & & \\
Dropped Group Electricity Cost Savings (\$millions) & & & \\
\hline Zero Persistence Assumption & 0.65 & 0.47 & \\
$\quad$ Standard Error) & $(0.03)$ & $(0.03)$ & $(0.06)$ \\
Observed Persistence & 1.75 & 1.39 & 2.57 \\
$\quad$ Standard Error) & $(0.07)$ & $(0.08)$ & $(0.2)$ \\
\hline \hline
\end{tabular}

Notes: This re-creates Table 7 over the projected lifetime of effects. Savings are extrapolated using the estimated linear decay parameter $\widehat{\delta}^{L R}$. Standard errors are calculated using the Delta method. 


\section{Appendix Figures}

Figure A1: Breakdown of Household Electricity Use

\section{US Household Electricity Use}

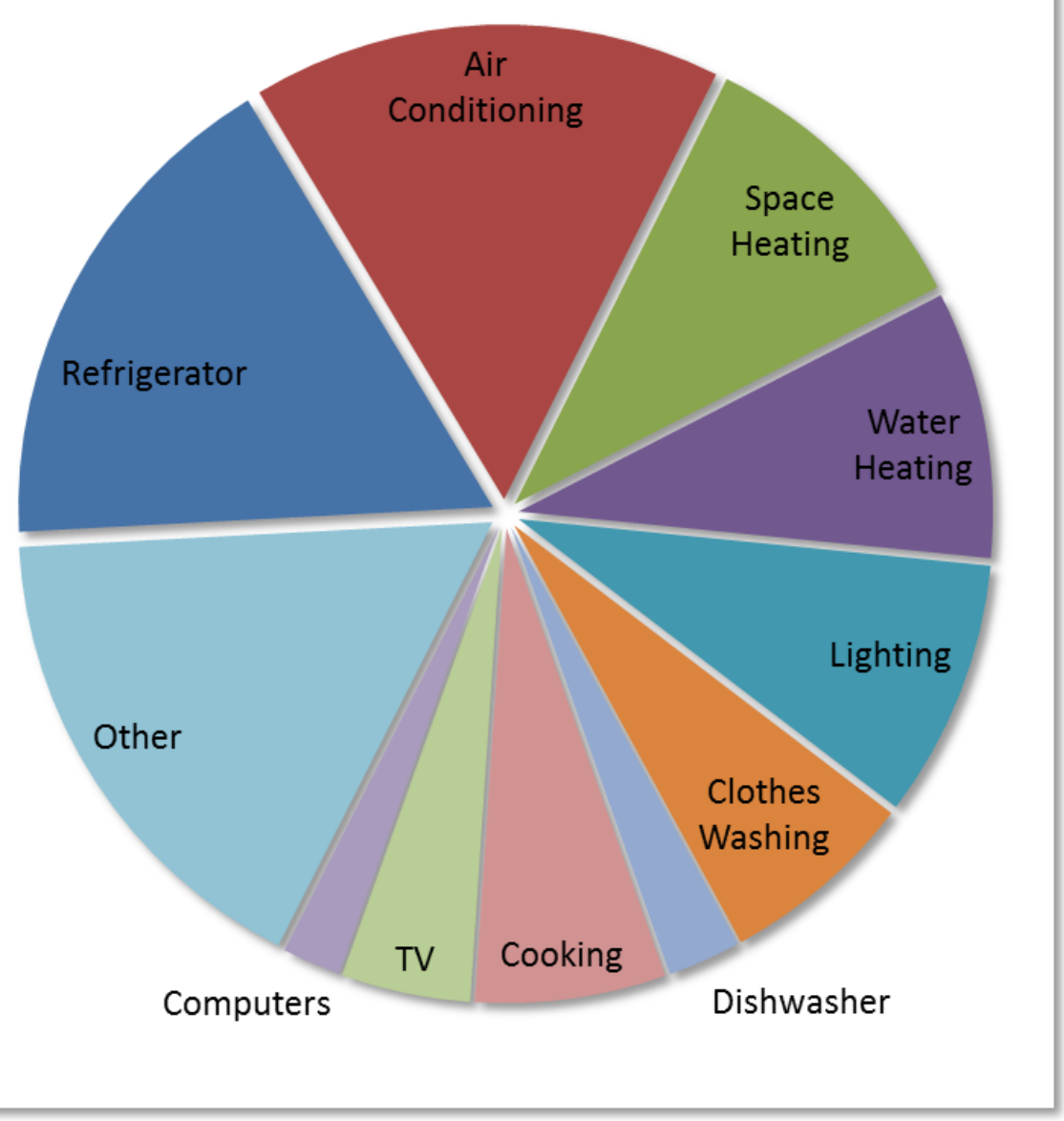

Notes: This figure shows the breakdown of electricity use for the average American household in 2001, the most recent year for which detailed figures are available. Source: U.S. Energy Information Administration (2009). 


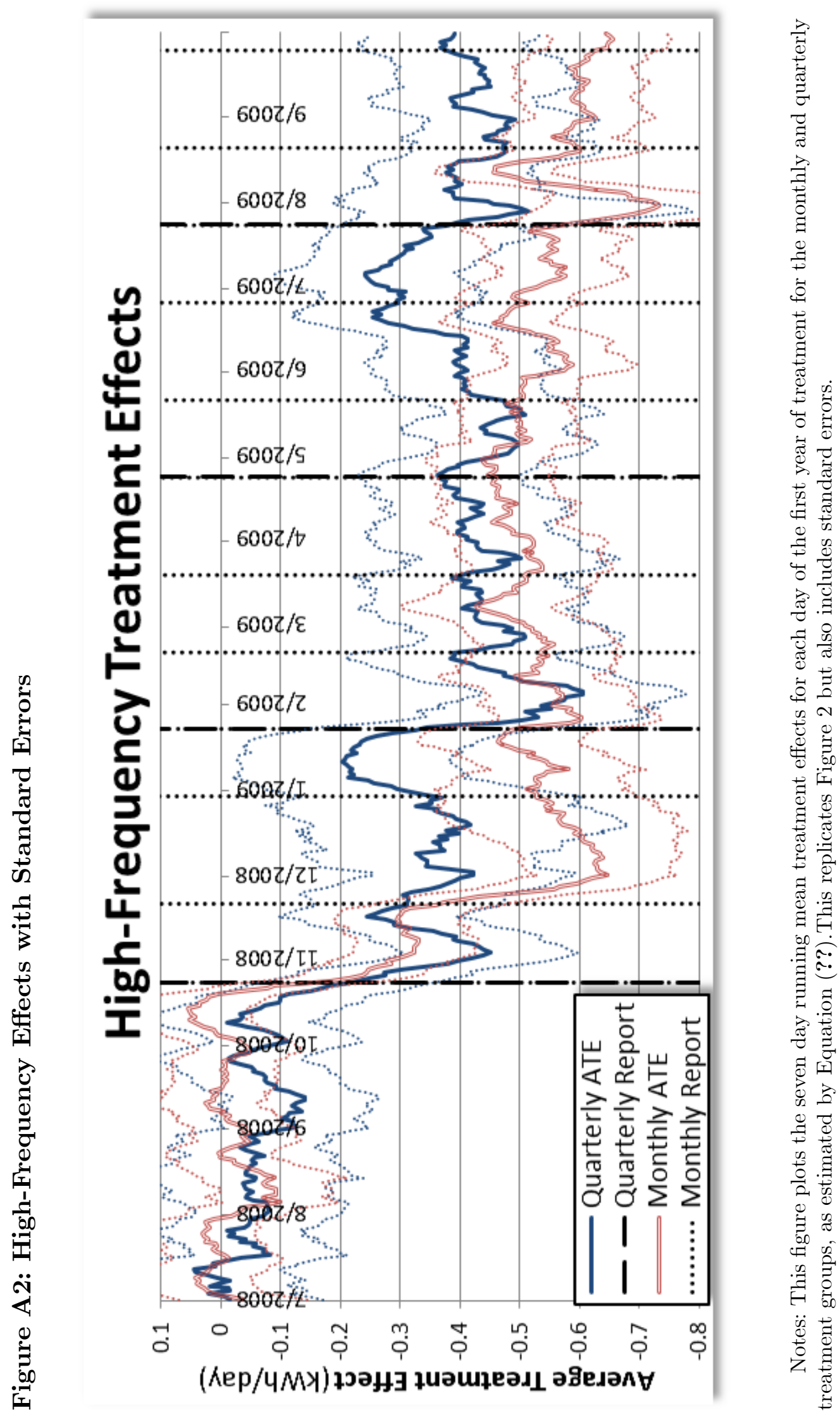

